\title{
Further Results Concerning the Simple Queueing Loss System
}

\author{
By ThOMAS L. VLACH, Michigan ${ }^{1}$ )
}

Eingegangen am 21. Juli 1969

\begin{abstract}
Summary: The output or departure process from a single channel queue with independent arrival intervals, exponential service times and no storage is considered. The main result obtained is that successive departure intervals are uncorrelated if and only if the arrival process is Poisson.

Zusammenfassung: Der output bzw. der Abgang eines einzelnen Wartekanals mit unabhängigen Ankunftsintervallen, exponentieller Bedienungszeit und ohne Speicherung wird untersucht. Als wesentliches Resultat ergibt sich, daß sukzessive Abgangsintervalle genau dann unkorreliert sind, wenn der AnkunftsprozeB Poisson-verteilt ist.
\end{abstract}

Schmid proves that in the simple queueing loss system $\mathrm{Gl} / \mathrm{G} / 1 / 0$ the output or departure process is a renewal process if and only if $\mathrm{GI}=M$, that is the arrival process is Poisson. In order to obtain this result, however, it must be assumed that the probability density functions of the arrival and service processes are both continuous and strictly positive on $[0, \infty]$. These restrictions eliminate from consideration a large class of important density functions including the gamma, uniform and deterministic.

We consider a less general queue, the $\mathrm{GI} / \mathrm{M} / 1 / 0$ and without the above restrictions prove that the departure process is uncorrelated if and only if $\mathrm{GI}=M$. We assume arrivals occur at epochs $\left\{t_{n} ; n \geq 0\right\}$. Let $Y_{n}=t_{n}-t_{n-1}, n>0$, $A(x)=P\left[Y_{n} \leq x\right] \forall_{n}, A(0+)=0$ and $a^{*}(\theta)=\int_{0}^{\infty} \mathrm{e}^{-\theta x} \mathrm{~d} A(x)$. Let $a_{j}(x)$ be the probability density function of the sum of $j$ consecutive arrival intervals, $j=1,2, \ldots$. The service times $\left\{S_{n} ; n \geq 0\right\}$ form a sequence of independent, identically distributed random variables independent of the arrival process with distribution function $B(x)=1-\mathrm{e}^{-\mu x}, b^{*}(\theta)=E\left[\mathrm{e}^{-\theta S_{n}}\right]=\frac{\mu}{\mu+\theta}$. Let $\left\{I_{n} ; n \geq 1\right\}$ be the sequence of idle periods that occur immediately after each departure, $F_{I}(x)=$ $P\left[I_{n} \leq x\right] \forall_{n}, f_{I}^{*}(\theta)=\int_{0}^{\infty} \mathrm{e}^{-x} \mathrm{~d} F_{I}(x)$. Let $\left\{U_{n} ; n \geq 0\right\}$ be the sequence of periods representing the elapsed time since the last arrival measured from the $n^{\text {th }}$ departure epoch, $F_{U}(x)=P\left[U_{n} \leq x\right] \forall_{n}$. Finally let $\left\{X_{n} ; n \geq 0\right\}$ be the sequence of departure intervals from the queue with $F(x)=P\left[X_{n} \leq x\right] \forall_{n}$.

$\left.{ }^{1}\right)$ Thomas L. VlaCH, Ph. D. University of Michigan, Dearborn, Michigan. 


\section{Theorem:}

The sequence $\left\{X_{n} ; n \geq 0\right\}$ is uncorrelated if and only if $\mathrm{GI}=M$, (Poisson arrivals).

\section{Proof:}

If $\mathrm{GI}=M$ then clearly the sequence $\left\{X_{n} ; n \geq 0\right\}$ is uncorrelated.

If the sequence $\left\{X_{n} ; n \geq 0\right\}$ is uncorrelated then $E\left[X_{n+1} X_{n}\right]=E\left[X_{n+1}\right] E\left[X_{n}\right]$, $n \geq 0$. But $E\left[X_{n+1} X_{n}\right]=E\left[\left(S_{n+1}+I_{n+1}\right) X_{n}\right]=E\left[S_{n+1}\right] E\left[X_{n}\right]+E\left[I_{n+1} X_{n}\right]$ since $S_{n+1}$ and $X_{n}$ are independent. Also $E\left[X_{n+1}\right] E\left[X_{n}\right]=E\left[S_{n+1}\right] E\left[X_{n}\right]$ $+E\left[I_{n+1}\right] E\left[X_{n}\right]$. Thus the condition for $X_{n}$ and $X_{n+1}$ to be uncorrelated is $E\left[I_{n+1} X_{n}\right]=E\left[I_{n+1}\right] E\left[X_{n}\right]$.

\section{From SCHMIDT}

$$
\text { [1] } F_{I}(x)=\int_{0}^{\infty} \int_{0}^{\infty} \frac{\mathrm{e}^{-\mu y} \mathrm{~d} A(y+w) \mathrm{d} w}{1-a^{*}(\mu)}
$$

and thus

$$
E\left[I_{n+1}\right]=\frac{\frac{\mu}{\lambda}-\left(1-a^{*}(\mu)\right)}{\left(1-a^{*}(\mu)\right)},
$$

and

$$
E\left[X_{n}\right]=\frac{1}{\mu}+E\left[I_{n+1}\right]=\frac{1}{\lambda\left(1-a^{*}(\mu)\right)}
$$

Finally,

$$
E\left[I_{n+1}\right] E\left[X_{n}\right]=\frac{\frac{\mu}{\lambda}-\left(1-a^{*}(\mu)\right)}{\mu \lambda\left[1-a^{*}(\mu)\right]^{2}} .
$$

In order to find $E\left[I_{n+1} X_{n}\right]$ we first find the joint probability density function of $I_{n+1}$ and $X_{n}$. Let $f(x, w)$ be the joint probability density function of $I_{n+1}$ and $X_{n}$ and let $g(y, w)$ be the joint probability density function of $U_{n}$ and $X_{n}$.

Then

$$
f(x, w)=\int_{0}^{w} \frac{a(x+y)}{1-A(y)} g(y, w) \mathrm{d} y .
$$

In order to find $g(y, w)$ let $g(y, w / t)$ be the conditional joint probability density function of $U_{n}$ and $X_{n}$ given $U_{n-1}=t, n \geq 1$. Then

$$
g(y, w / t)=\frac{[1-A(y)] \mathrm{e}^{-\mu y}}{1-A(t)}\left\{a(w-y+t)+\int_{0}^{w-y} \mathrm{e}^{-\mu \xi} a(w-y-\xi+t) \sum_{j=1}^{\infty} a_{j}(\xi) \mathrm{d} \xi\right\}
$$

and

$$
g(y, w)=\int_{0}^{\infty} g(y, w / t) \mathrm{d} F_{U}(t)
$$


But

$$
\begin{aligned}
F_{U}(t) & =P\left[U_{n} \leq t\right]=P\left[S_{n} \leq t, S_{n}<Y_{n+1}\right] \\
& +P\left[S_{n} \leq Y_{n+1}+t, Y_{n+1}<S_{n}<Y_{n+1}+Y_{n+2}\right] \\
& +P\left[S_{n}<Y_{n+1}+Y_{n+2}+t, Y_{n+1}+Y_{n+2}<S_{n}<Y_{n+1}+Y_{n+2}+Y_{n+3}\right] \\
& +\cdots=\int_{0}^{t} \mu \mathrm{e}^{-\mu y}[1-A(y)] \mathrm{d} y+\int_{0}^{\infty} \int_{0}^{t} \mu \mathrm{e}^{-\mu\left(y_{1}+y_{2}\right)}\left[1-A\left(y_{1}\right)\right] \mathrm{d} y_{1} \mathrm{~d} A\left(y_{2}\right) \\
& +\int_{0}^{\infty} \int_{0}^{\infty} \int_{0}^{x} \mu \mathrm{e}^{-\mu\left(y_{1}+y_{2}+y_{3}\right)}\left[1-A\left(y_{1}\right)\right] \mathrm{d} y_{1} \mathrm{~d} A\left(y_{2}\right) \mathrm{d} A\left(y_{3}\right) \\
& \int_{0}^{t} \mu \mathrm{e}^{-\mu y}[1-A(y)] \mathrm{d} y \\
& \frac{1-a^{*}(\mu)}{}
\end{aligned}
$$

Combining (2), (3) and (4),

$$
\begin{gathered}
E\left[\mathrm{e}^{-\theta_{1} I_{n+1}} \mathrm{e}^{-\theta_{2} X_{n}}\right]=\int_{0}^{\infty} \int_{0}^{\infty} \int_{0}^{w} \mu^{2} \frac{\mathrm{e}^{-\theta_{1} x} \mathrm{e}^{-\theta_{2} w} a(x+y)}{1-a^{*}(\mu)} . \\
\left\{\int_{0}^{\infty} \mathrm{e}^{-\mu(y+t)} a(w-y+t) \mathrm{d} t+\int_{0}^{\infty} \int_{0}^{w-y} \mathrm{e}^{-\mu(\xi+y+t)} a(w-y-\xi+t) .\right. \\
\left.\sum_{j=1}^{\infty} a_{j}(\xi) \mathrm{d} \xi \mathrm{d} t\right\} \mathrm{d} y \mathrm{~d} x \mathrm{~d} w
\end{gathered}
$$

which upon simplification reduces to

Then

$$
\frac{\mu^{2}\left[a^{*}\left(\theta_{2}\right)-a^{*}(\mu)\right]\left[a^{*}\left(\theta_{2}+\mu\right)-a^{*}\left(\theta_{1}\right)\right]}{\left(\mu-\theta_{2}\right)\left(1-a^{*}(\mu)\right)\left[1-a^{*}\left(\mu+\theta_{2}\right)\right]\left[\theta_{1}-\left(\theta_{2}+\mu\right)\right]} .
$$

$$
\begin{aligned}
E\left[I_{n+1} X_{n}\right] & =\left.\frac{\partial^{2}}{\partial \theta_{1} \partial \theta_{2}} E\left[\mathrm{e}^{-\theta_{1} I_{n+1}} \mathrm{e}^{-\theta_{2} X_{n}}\right]\right|_{\theta_{1}=\theta_{2}=0} \\
& =\frac{-\frac{\mu^{2}}{\lambda} a^{* \prime}(\mu)-\left[1-a^{*}(\mu)\right]^{2}+\frac{\mu}{\lambda}\left[\frac{\mu}{\lambda}-\left(1-a^{*}(\mu)\right)\right]}{\mu^{2}\left[1-a^{*}(\mu)\right]^{2}} .
\end{aligned}
$$

Equating (5) with (1) and simplifying results in the Ricatti type differential equation

$$
\frac{\mu^{2}}{\lambda} a^{* \prime}(\mu)+\left[1-a^{*}(\mu)\right]=0
$$

whose solution is $a^{*}(\mu)=\frac{\lambda}{\lambda+\mu}$ which implies arrival intervals have a negative exponential distribution with parameter $\lambda$.

\section{Bibliography}

ScHMIDT, G.: Über die in einem einfachen Verlustsystem induzierten stochastischen Prozesse, Unternehmensforschung, 11,1967 , pp. 95-110. 